\title{
Time To Discharge and Its Predictors Among Admitted Patients in Felege Hiwot Comprehensive Specialized Hospital North-West Ethiopia, a Prospective Cohort Study
}

Afework Orcho ( $\square$ afeworkhailu866@gmail.com ) Jigjiga University

Gebretsadik Keleb

Jigjiga University

\section{Tewodros Getnet}

Dire Dawa University

\section{Research Article}

Keywords: admission, discharge time, inpatients, Ethiopia

Posted Date: December 28th, 2021

DOI: https://doi.org/10.21203/rs.3.rs-1152268/v1

License: (9) This work is licensed under a Creative Commons Attribution 4.0 International License.

Read Full License 


\section{Abstract \\ Background}

Hospital long stay is a serious social and economic problem, leading to elevated medical expenses, increased workload, and financial burden. Patients' discharge time was different in admitted patients in hospitals. In Ethiopia, there was limited evidence why the patients' length of stay is different in roughly the same condition.

\section{Objective}

This study aimed to determine a time to discharge and its predictors among admissions in Felege Hiwot Hospital in Bahir Dar, Northwest Ethiopia, 2020 G.C

\section{Methods}

Prospective cohort study was conducted among 812 systematically selected study participants. The data collected using standardized questionary was entered into EPI DATA and exported into STATA for analysis. Appropriate descriptive statistics were stated. Incidence density to estimate time to discharge, and life table and Kaplan Meier curve to determine the cumulative survival of patients in the wards were used. Cox proportional hazard (Weibull) model was used to examine predictors of discharge. Variables from bivariate analysis with $p<0.25$ were a candidate for the final model. Adjusted Hazard Ratio with their $95 \% \mathrm{Cl}$ at $p<0.05$ was used to predict discharge. Model fitness was checked.

\section{Results}

A total of 812 admitted patients participated in the study giving a total response rate of $95 \%$. Fifty-five presents were females; the median age was 32 . The total times at admission wards in medical were 1827 and in surgical 2050 days. The incidence densities of discharge in medical and surgical wards were $361 / 1827$ and 359/2050-person days respectively. The median time to discharge of all participant admission was four days. Predictors of discharge were age (AHR 1.04(95\% $\mathrm{Cl} 1,02-1.06)$ ), family care (AHR .69(.51-.94)), admission source (AHR 2.07(1.39-2.90)), admission time (AHR .57(.49-.67)). Lab.result delay (AHR .67(.55-.81)), radiological result delay (AHR .78 (.64-.95)), presence of comorbidity (AHR.50(.42-.60)), disease burden group (AHR 2.15(1.84-2.66)), specialty (AHR 0.66 (0.56-0.78)), nurse-topatient ratio (AHR 1.95 (1.57-2.43)), and medication adherence (AHR 2.83(2.29-3.51).

\section{Conclusion}


Surgical admitted patients discharged in longer time than medical. It is important to emphasize good communication of respective specialties on the lab. and radiology result test waiting time and also teaching the benefit of medication adherence to the patients.

\section{Background}

Hospitals are a vital part of a health system that in coordination with other parts, provides health to the community. Hospitals have an important role in providing health services; consequently, they have a major impact on the efficiency of a health system. The most important tool for evaluating hospital performance is hospital indicators. Among these indicators, length of stay (LOS) in a hospital is one of the most important indicators that are widely used today to measure the efficiency of hospitals $(1-3)$.

Time to discharge is the total spent time of patients from admission to discharge. It is one of the important indicators of health service management. The appropriate time to discharge as ideal time in each department varies from country to country. Inwards except emergency, which is 24 hours for any case, the ideal timeliness of hospital stay in hospitalized patients is the average length of stay in respective wards. It is measured as an average number of days from admission to discharge for each inpatient $(2,4)$.

Hospitals are struggling to provide timely care to a progressively increasing number of visits to the facility. The government of Ethiopia health minister also established hospital reform implementation guidelines in 2010 to measure quality improvement. In which each hospital should have a liaison and referral service that is responsible for managing hospital bed management, facilitating ward admission, and providing social service support to different case teams. To achieve these quality improvements the guideline briefed no patient should remain in hospital any longer than necessary time due to irregular ward rounds, delay in arranging necessary follow up appointments, discharge or referral papers, lack of hospital administrative or cashier functions to handle discharges 24 hours a day, 365 days a year (3).

Discharge time in the hospital is varied according to the patient's variation during a presentation to and hospital process. But in some cases, the variation is beyond the expected length of stay in hospitals. It is worldwide problems that burden in different departments of hospital reported that activities ware not gone as scheduled. Patients stay longer in admission beyond the scheduled time due to different reasons. Most problems or stresses observed in the public hospital system of New South Wales find expression, in one form or another, be it a lack of senior doctors, ineffective bed management practices, the widespread use of locums, or poor communication with patients or their attendants (5). Length of hospital stay was longer in the patients unable to gain access to an appropriate hospital inpatient bed than those who gain access to an appropriate hospital inpatient bed which is also linked to increased waiting time for medical care in the health facility(6).

In Ethiopia where health resources are limited and underdevelopment is a crucial problem, patients in hospitals complain about long stays which is a serious social and economic problem, leading to elevated medical expenses as well as high workload and financial burden to hospitals (7). 
Quality management and improvement to advance the quality of health service delivery by health facilities across the country, remarked positive improvements, including the reduced outpatient waiting time, reduced institutional mortality rate, bed occupancy, the average waiting time for surgery, and patient satisfaction. However, no reports seen in the country on inpatients' admission improvements (8).

Despite the unclear relationship between longer hospital stay and quality of care, a longer hospital stay is associated with the financial burden of both hospitals and patients leading to overall raise in health care costs of the county. Everyone agrees that prolonged hospital stay due to emergency and inpatient boarding is unsafe; is associated with excess deaths, decreases hospital efficiency, and makes the lives of clinicians difficult, resulting in dissatisfaction and burnout.

It is also associated with complications like nosocomial infections, immobility, pressure sores, deep vein thrombosis, deconditioning, and then worsening patient's quality of life in inpatient admission. Which also requires an extra cost to get treated and disturbs the efficiency of services. Longer stay of hospitalized patients, their discharge pattern, and admission rate were described to decrease acute bed availability leading to overcrowding and hospital utilization blockage (9-14).

Annual admission rate in Ethiopia mental health care facility reported 4 in 100000 population with 63 days' median length of hospital stay(15). To have quality health care including inpatient in hospitals, the government finances an enormous amount of budget and oversee hospital operations by establishing hospital key performance indicator(4).

The hospital-related factors such as the inability of patients to access hospital beds, delayed laboratory tests, radiological results, and consultation delay were associated with a longer stay and the major issue currently facing hospitalized patients as a worldwide problem. These are some of the factors that lead to a long stay in hospital in many different pieces of literature. During the last several years, it has been reported prominently in Australia, Northern America, and Europe.

However, longer stay in hospital may not be exclusively in emergence department problem in Ethiopia. It might be a problem of wards admitted patients which may be affected by facility, patient, and disease characteristics as well. Study to reason out long stay in hospitals is a few in Ethiopia such health resource-limited settings. And also reported studies of the extent of hospital stay vary between studies due to restriction of study to particular wards or age groups and types of methodology used.

Questions are raising why some patients stay longer and some shorter duration even though in similar conditions like the same case, ward, admission or beyond average length of stay. Little is known about the causes of variation in lengths of stay at the different departments and referral hospitals in Ethiopia, principally because of the shortage of visibly available data about patients' length of stay.

Therefore, this study was searched for median discharge time, survival, and factors predicting discharge time among admitted patients. Interest in the quality of health care has increased by health care professionals and policymakers due to the perceived need for continuous quality improvement and 
transparency to the public. Hence, it is important to get insight into the quality of health care indicators such as patient length of stay in hospitals.

This study intends to establish factors related to the facility, patients, and disease characteristics that can explain the length of hospital stay. Interests are growing on policymakers and health professionals to predict the time to discharge and then optimize the use of hospital resources. Because of, increasing demand of service users globally and nationwide on quality health care, it is important to examine problems that can be modifiable regarding service areas and patients' related characteristics of the length of hospital stay.

Hospital managers and policymakers design systems that allow services to be performed more efficiently through the identification of predictors of time to discharge which may represent the potential target for interventions. It also tries to investigate whether patients stay longer due to clinical implications or perhaps due to non-clinical reasons. In addition, knowledge of the predictors to timely discharge benefits users regarding expectations of stay in terms of cost, time, and other resources. Furthermore, it would try to disclose information for future research on predictors of hospital stay.

\section{Methods And Materials}

\section{Study setting}

The study was conducted from July to August in 2020 at Felege Hiwot comprehensive specialized hospital which is found in Bahir Dar city. Bahir Dar is the capital city of Amhara national regional state and is found $563 \mathrm{~km}$ northwest of Addis Ababa. The hospital was established in 1963 G.C. and is located at the northern end of the city near Lake Tana. It is intended to serve five million people in the region. Currently, the hospital delivers health services as outpatient and inpatient management in different departments.

The hospital has 482 beds in 11 different specialized cares which has one emergency ward and Inpatient wards such as Gynecological \&Obstetric, Surgical, orthopedics, Medical, Pediatric, L\&D, Eye unit, NICU, psychiatry, oncology, and 22 OPDS and. Inpatient admission roots were from emergency, OPD, and ICU. In the 2011 annual report admission of 25962 in all wards (unpublished reports).

For purpose of this study, inpatient wards were classified into two major wards named medical wards and surgical wards. Medical wards included in this study were the adult medical ward, pediatrics, and oncology. Under Surgical wards general surgical ward, orthopedics, gynecology and, labor and delivery were categorized $(21,22)$.

A prospective cohort study that has a better ability to access missed information from registrations to determine hospital stay was used. Out of 854 respondents, 812 respondents each followed for a maximum of fourteen days from July to August in 2020.

\section{Study participant}


All admissions from July to August in 2020 were the source population for the study. Patients admitted in general surgical, orthopedics, gynecology, labor and delivery, adult medical, pediatrics, and oncology wards admitted patients in the study period from July to August in 2020 at Felege Hiwot Comprehensive specialized hospital was being candidates for the study.

Inclusion criteria: Patients admitted in general surgical, orthopedics, gynecology, labor and delivery, medical wards include adult medical, and pediatrics and oncology wards admitted patients in the study period.

Exclusion criteria: admitted patients moved from one study ward to other study wards (staying in different study wards was terrible to determine how long stayed in admission) were excluded.

The Selected individual in the ward was followed daily in inpatients for the follow-up variables and recorded according to the structured questionary.

Events: discharged.

Censored: loss to follow-up, transferred, died, and not discharged in the data collection period

Epi info version 7.2.0.1 was used to calculate the maximum sample size by considering $95 \%$ confidence interval, power of 80 , and 1:1 ratio of medical and surgical ward patients. The proportion of discharge in 14 days stay was $50 \%$ in internal medicine wards and a $10 \%$ difference in surgical ward admission was taken due to lack of similar study done in Ethiopia. The total sample size calculated for medical and surgical ward patients each was 388 , gave the total sample 776 , and $10 \%$ added to compensate lost follow up and drop out of patients, and the final sample size became 854 .

\section{Sampling technique/procedure}

Systematic random sampling was used to select patients in both groups. The total patients flow from the previous year during the same period was used to calculate the sampling interval. From each medical ward every other and from each surgical ward every third admission was selected (Figure 1).

\section{Study variables}

\section{Dependent variable}

Time to discharge

\section{predictors of discharge}

The patient's characteristics include: Sex Age, marriage, Occupation, Insurance status, Education, Family care.

Facility characters include: delay of lab results, delay of radiological results, specialty, admission time, sources of admission, nurse to patient's ratio, physician to patient's ratio 
Disease character includes: Diagnosis, Comorbidity, Disease burden group, Nosocomial infections, Number of medicines, Medication adherence, Readmission in a month.

Operational definitions/measurements

- Time to discharge: in this study, time taken from admission till discharged from inpatients to home or other facilities in the follow-up period.

- Discharge: discharging of patient within14 days in admission to a home or other facility. To measure inpatient service efficiency, hospitals use two-week duration data from patients. So, this paper uses a two-week duration for each patient to gather almost similar information from patients (4).

- The primary diagnosis is the main diagnosis for admission (10).

- Nosocomial infection: presences of hospital-acquired infection registered on patients' card in time to discharge.

- Disease burden group: in this study primary diagnoses were categorized as the global burden of disease into four categories; communicable, non-communicable, injuries and maternal, perinatal conditions.

- Delay in lab/radiological results; any result report comes after its usual time which is based upon inquiry from laboratory/radiology staff about the time taken by each inpatient investigation (23).

- Comorbidity; the presence of more than one diagnosis.

- Number of medications; a number of medicines each patient taking and registered in patients' card.

- Time of admission; off and office our admission. Those admitted at 8:30 am to 5:30 pm from Monday to Friday excluding Holydays are office hour admission. And other ways off-hour admission.

- Family care; the presence of an attendant during the stay period.

- Nurse to patients ratio; the number of nurses divided by the number of patients at admission.

- The physician to patients ratio; the number of physicians divided by the number of patients at admission.

- Medication adherence; using the Morisky scale 4 questions scores were given from 0-4 according to the answer by each patient. It is a validated tool in researches $(24,25)$.

Morisky Scale Scoring system

1. Do you ever forget to take your medicine?

2. Are you careless at times about taking your medicine?

3. When you feel better, do you sometimes stop taking your medicine?

4. Sometimes if you feel worse when you take the medicine, do you stop taking it?

Yes $=0$ and $\mathrm{No}=1$

- Zero is the lowest level of medication adherence 
- 4 is the highest level of medication adherence. Patients who scored 0-2 were non-adherent. while 3-4 scored were adherent.

\section{Data collection instrument and process}

Data were collected by trained collectors prospectively using structured questionary by interviewing participants and from medical records. The questionary was derived from related literature. All candidate patients were registered and given an ID with questionary bearing baseline data. Three-degree nurses who had a day of training on how to record data filled the questionaries.

The baseline data like age, sex, primary diagnosis, and date of admission were recorded from the patient card while education, insurance status, marriage, and occupation short interview at admission were carried out as soon as the patient was admitted. Laboratory and radiological investigations report that came after turnaround time was not applicable to record as delay per each investigation turn round time. Since investigation results were so late than turn round time, even investigation stay more than days in ward admitted patients and no results reports were available at its usual time. So, this study used the number of days' investigation reported back to the patients were recorded. For nosocomial infection followed presences of hospital-acquired infection registered on patients' cards is recorded in follow up period. Comorbidity was recorded from cards if more than one diagnosis presents. Medication adherence using the Morisky scale, each participant was asked 4 questions given a score from 0-4.

\section{Data quality}

The questionary was pre-tested in other similar hospital study areas on $5 \%$ of the sample to overcome any difficulties in variables measuring during data collection and any further valid and reliable ways to measure. The principal investigator had close supervision to overcome any mistakes from data collectors. After checking all questionary for consistency and completeness the data collectors submitted the filled checklist to the principal investigator. Incorrectly filled or missed records were sent back to the respective data collector for correction. Data collectors had no information on which ward was related to outcome variables to reduce bias.

\section{Statistical methods}

The collected data were coded, cleaned, and entered into EpiData Version 3.1, and exported into STATA version 15 for data processing and analysis. The first analysis was descriptive statistics to examine the proportion of participants who responded. Then the median and range of age were determined after checking the normality. Using person-days, the incidence density of discharge was determined. The cumulative probability of time to the discharge was determined by the life table and Kaplan Meier survival curve. A Log-rank test to compare survival of medical and surgical wards was used. Variables from bivariate analysis with $p<0.25$ were candidates for the final model. The final model was determined by the forward variable selection method with $p<0.05$. Continuous variable, age was checked for its log linearity by grouping and fractional polynomial method, and each methods model was compared using 
the list Akaike information criterion (AIC) value, model. Age, as a continuous log hazard with list AIC, was selected, and for meaning full interpretation 5-year increase was used. Nurse-to-patient ratio and medication adherence data were grouped according to previous literature. The final model was checked for hazard proportional assumption using global test and violated, so a farther parametric survival model with list AIC (Weibull hazard) was used. Post-estimation plots for the Weibull distribution assumption were checked for model fitness. Adjusted Weibull Hazard risk with their $95 \% \mathrm{Cl}$ and $\mathrm{p}<0.05$ was used to identify the predictors of discharge.

\section{Results}

\section{Population profile}

A total of 812 admitted patients participated in the study giving a total response rate of $95 \%$. Five percent (19 from medical and 23 from surgical wards) refused the consent at admission. Of the participants 55 present were females and about $43 \%$ were illiterate. Participants' age ranges from one month to 88 years with a median age of 32 years.

In the medical ward, half of the study participants were males and $41 \%$ of the study participants were more than 40 years old. About forty percent were illiterate. In the surgical ward, about fifty-nine percent of the study participants were female and $18 \%$ of the study participants were less than 20 years old. Fortysix percent were illiterate (Table 1). 
Table 1

Socio-demographic characteristics of admitted patients in Felege Hiwot compressive specialized hospital, $2020(n=812)$

\begin{tabular}{|c|c|c|c|}
\hline \multirow[t]{2}{*}{ variables } & & \multicolumn{2}{|c|}{ Surgical and medical ward pts } \\
\hline & & Frequency & Percent (\%) \\
\hline \multirow[t]{4}{*}{ Age group } & $0-20$ & 191 & 23.52 \\
\hline & $21-30$ & 208 & 25.62 \\
\hline & $31-40$ & 175 & 21.55 \\
\hline & $40+$ & 238 & 29.31 \\
\hline \multirow[t]{2}{*}{ Sex } & Male & 364 & 44.83 \\
\hline & Female & 448 & 55.17 \\
\hline \multirow[t]{4}{*}{ Education } & Illiterate & 351 & 43.23 \\
\hline & Primary & 184 & 22.66 \\
\hline & Secondary & 202 & 24.88 \\
\hline & Tertiary & 75 & 9.24 \\
\hline \multirow[t]{2}{*}{ Marital status } & Married & 477 & 58.74 \\
\hline & Single & 335 & 41.26 \\
\hline \multirow[t]{2}{*}{ Insurance } & Insured & 409 & 50.37 \\
\hline & Uninsured & 403 & 49.63 \\
\hline \multirow[t]{2}{*}{ Family care } & Present & 766 & 94.33 \\
\hline & Absent & 46 & 5.67 \\
\hline
\end{tabular}

\section{Survival status of admitted patients}

The total time admitted patients followed was 3877 days. The incidence density of discharge was $720 / 3877$ person-days. One-fourth, $50 \%$ and $75 \%$ of the admitted patients were discharged on days 3,4 and 6 respectively (Table 2 and figure 2). The discharge time was longer for surgical wards admitted patients compared to medical ward admitted patients (figure 3). 
Table 2

Life table for discharge of admitted patients in Felege Hiwot compressive specialized hospital 2020 ( $\mathrm{N}=812$ )

\begin{tabular}{|llllll|}
\hline Interval & Beg. Total & discharges & Censored & C.Survival & (95\% conf.int) \\
\hline $1-2$ & 812 & 70 & 12 & 0.9132 & $0.8915-0.9306$ \\
$2-3$ & 730 & 76 & 10 & 0.8174 & $.7889-0.8425$ \\
$3-4$ & 644 & 128 & 16 & 0.6529 & $0.6185-0.6850$ \\
$4-5$ & 500 & 126 & 8 & 0.4871 & $0.4514-0.5218$ \\
$5-6$ & 336 & 121 & 9 & 0.3240 & $0.2909-0.3575$ \\
$6-7$ & 236 & 73 & 4 & 0.2229 & $0.1937-0.2535$ \\
\hline $7-8$ & 159 & 43 & 3 & 0.1621 & $0.1364-0.1897$ \\
\hline $8-9$ & 113 & 23 & 3 & 0.1286 & $0.1054-0.1543$ \\
\hline $9-10$ & 87 & 18 & 0 & 0.1020 & $0.0811-0.1256$ \\
\hline $10-11$ & 69 & 8 & 1 & 0.0901 & $0.0704-0.1127$ \\
\hline $11-12$ & 60 & 15 & 1 & 0.0674 & $0.0503-0.0877$ \\
\hline $12-13$ & 44 & 11 & 1 & 0.0504 & $0.0357-0.0686$ \\
\hline $13-14$ & 32 & 6 & 1 & 0.0408 & $0.0276-0.0577$ \\
\hline $14-15$ & 25 & 2 & 23 & 0.0347 & $0.0219-0.0520$ \\
\hline
\end{tabular}

Table 3

The log-rank test of medical and surgical wards

\begin{tabular}{|lll|}
\hline wards & Events observed & Events expected \\
\hline Medical wards & 364 & 333.21 \\
\hline Surgical wards & 356 & 386.79 \\
\hline Total & 720 & 720.00 \\
\hline Chi2 $=6.96 p<0.000$ & \\
\hline
\end{tabular}

The survival function (time to discharge) of medical and surgical wards was significantly different (Table $3)$.

\section{Predictors of Discharge}

The variables included in the final model after forward selection $(p<0.05)$ were age, presence of family care, admission source, admission time, lab. result delay, radiological result delay, disease burden group, 
presence of comorbidity, specialty, nurse to patients' ratio, and medication adherence (Table 4).

In this study, the discharge time was longer in children, i.e., as the age of the patient increase by 5 years, the length of stay of admitted patients decreased by 4\% (AHR 1.04(1.02-1.06)). Radiology result arrival more than one-day delay increased time to discharge by $22 \%$ (AHR .78 (.64-.95)). The presence of comorbidity increased discharge time by $50 \%$. (AHR.50(.42-60)). Admission from emergency decreased discharge time by about 2 folds (AHR 2.07(1.39-2.90)). Admission from OPD decreased discharge time by about 1.6-fold (AHR 1.59 (1.08-2.33))

Discharges of off-hour admission increased length of stay by 43\% (AHR .57(.49-.67)). Discharge of combined non-communicable, maternal and prenatal condition admission was decreased discharge time by 2.2 times compared to discharge of combined infectious and injuries admission (AHR 2.15(1.842.66)). The discharge time of the surgical ward was increased by $34 \%$ (AHR .66(.57-.78)). In this study, patients with family care showed a $31 \%$ higher length of stay (AHR .69(.51-.94)).

Patients with a lab result arrival second or latter day discharged time increased by $33 \%$ (AHR $.67(.55-81)$ ). At patient admission nurse to patient ratio $\geq 1: 2$ decreased discharge time by two-fold (AHR 1.95 (1.57-2.43)). In addition, patients with medication adherence decreased length of stay by 2.8 -fold (AHR 2.83(2.29-3.51)). 
Table 4

Predictors of discharge time in Felege Hiwot compressive specialized hospital,2020

\begin{tabular}{|lllll|}
\hline Variables & CHR & AHR & P-value & $95 \%$ Cl AHR \\
\hline Radiology result delay & $.35(.29-41)$ & 0.78 & 0.012 & $0.64-0.95$ \\
\hline Comorbidity yes & $.37(.32-.44)$ & 0.50 & 0.000 & $0.42-0.60$ \\
\hline Admitted from & & & & \\
\hline Emergency & $2.92(2.04-4.19)$ & 2.01 & 0.000 & $1.39-2.90$ \\
\hline OPD & $2.20(1.52-3.19)$ & 1.59 & 0.017 & $1.08-2.33$ \\
\hline Admission time: & $.47(.40-.55)$ & 0.57 & 0.000 & $0.49-0.6$ \\
off hr. admission & & & & \\
\hline DiseaseBurdenGroup & $3.2(2.67-3.80)$ & 2.22 & 0.000 & $1.84-2.66$ \\
\hline Specialty: Surgical wards & $.88(.76-1.02)$ & 0.66 & 0.000 & $0.56-0.78$ \\
\hline Family care & $.78(.58-1.05)$ & 0.69 & 0.017 & $0.51-0.94$ \\
\hline Lab. result delay & $.44(.38-.51)$ & 0.67 & 0.000 & $0.55-0.81$ \\
\hline Age/5 & $1.02(1.00-1.04)$ & 1.04 & 0.000 & $1.02-1.06$ \\
\hline Nurse/patient & $2.80(2.32-3.36)$ & 1.95 & 0.000 & $1.57-2.43$ \\
\hline Medication adherence & $4.25(3.44-5.25)$ & 2.83 & 0.000 & $2.29-3.51$ \\
\hline
\end{tabular}

The Post-estimation plot of hazard function shows increasing of discharge through time but not increasing and decreasing of discharge through time (figur4). Also, estimated cumulative hazards plotted against the Cox-Snell residuals are close to the reference line (figure 5).

\section{Discussion}

In this study incidence density to be discharged in 14 days was $720 / 3877$ person-days. This means if we follow 3877 admissions for one day 720 patients would be discharged. The incidence density of discharge in medical wards were 364/1803 i.e., if we follow 1803 admitted patient in the medical ward for one day 364 patients would be discharged. Fifty percent of the medical ward were discharged within five days while in surgical within four days. Even though surgical ward admission stays longer, the median discharge time is shorter than medical.

In this study, as the age of the patient increase by five years, discharge time length shortened by $4 \%$. This finding opposes researches finding which showed the probability of experiencing prolonged discharge from the hospital is significantly associated with increasing age $(1,26)$. In this study population, older 
patients were more admitted with diseases that take a shorter time to discharge like conditions gynecological and oncological problems and also it included broad age ranges than other studies. This study is important for consideration and seeking attention for young ages, being admitted for a longer duration, to have sufficient resource allocation bearing in mind the limitation of the studies. In this study, patients with family care showed a $29 \%$ increase in length of stay compared to patients without family care. This finding is inconsistent with findings from England national health service on length of hospital stay, in case of absence of family care a patient cannot be discharged home until he/she can manage independently or patients without adequate care at home stay longer for social reasons than medical reason $(13,27)$. Attendants of patients are key decision-makers. So, the absence of such a person therefore may affect finding a suitable place. Further research is needed on why this statistical association exists in family care and the long time to be discharged. This finding is important in the understanding of how family care affects hospital stay.

In this study patients with comorbidity showed $50 \%$ increases in discharge time length. This is confined to the study from England and New Zealand (28-30). This is due to the presence of concomitant problems requiring further treatment time leads to longer than the average length of the hospital (29). The significance of the medical comorbidities in this study may suggest that length of stay in admitted patients with comorbidity may not be further modifiable, but it may be more appropriate for future interventions to target such conditions to reduce longer time hospitalization.

The discharge time of combined non-communicable, maternal and prenatal condition admission was 2.2 times longer than the discharge of combined infectious and injuries admission. Gynecology and obstetrics length of stay in Felege Hiwot Comprehensive Specialized Hospital is shorter than other wards and also orthopedics admitted patients stayed longer to be discharged which can contribute to discharge time difference in two wards (20). This evidence is important to emphasize hospital stay in these disease groups. However, individual case severity and other conditions should be considered. Patients with medication adherence discharged were 2.8 times shorter than non-adherent patients. This is similar to findings from research findings in Indian which showed good medications adherence has a favorable effect on health outcomes including reducing the length of hospital stay(31). Even though this study was limited to determining individual health conditions, patients with good medication adherence benefited from early discharge.

The discharge time of surgical wards was $34 \%$ higher than the medical ward. Although the discharge time variation in the ward exists, no established reason for this difference. A possible explanation for this variation is an admission of elective surgical cases which had longer hospital stay time than other ward admissions in case of this hospital annual report depicts (20). This evidence is important to realize that surgical patients are admitted for a longer duration and should be considered even there are hidden factors in this study.

Radiology result arrival more than one-day increased discharge time by $22 \%$ compared to first-day arrival admissions. Similarly, Patients with a lab result arrival second or latter-day discharge time increased by 
$34 \%$ compared to laboratory result arrival on the first day. This finding is consistent with findings from south Ethiopia and Brazil which states waiting time for radiological service and waiting time for laboratory results significantly affected hospital discharge time $(7,32)$. It is obvious that whenever there are delayed test results then medical treatment and decision would wait a long time. Awaiting the results of tests for the care team to make clinical decisions was responsible for the majority of extra time spent in the hospital (32). It is important to notice that each investigation results reported at their scheduled turnaround time to ensure the effectiveness of hospital performance thereby maintaining length of hospital stay(4).

Admission from emergency decreased discharge time by about 2-fold, while from OPD decreased by 1.6fold compared to ICU admission. This finding is similar to research which showed that patients admitted through emergency have a significantly shorter length of stay as compared to those from other admission roots. Outpatient admissions were planned and significantly related to the higher need for instrumental and diagnosis exam which tend to prolong length of hospital stay (33). This finding is also important to understand admission sources are important determinants of discharge time and should be investigated how it determines considering drawbacks of this study.

The discharge time of admission for off-hour admission was $43 \%$ increase compared to the discharge of office hour admission. This finding is similar to studies from Australia $(6,34)$. Possibly due to off-hour admissions were found to have a somewhat higher length of stay due to decreased quality of care including increased medication error rates, workload, and low intensity of inpatient care (35). Even though individual disease severity is different, this study result is important evidence to show admission time variation will determine discharge time length, and consideration of off-hour admissions care quality is vital.

Patients admitted to nurse-to-patient ratio $\geq 1: 2$ discharged in 2 times lower length of stay than nurses to patient ratio $<1: 2$ at admission. This result is consistent with findings of studies that stated increased nurses per patient significantly reduced length discharge from hospitals $(36,37)$. This is evidenced in the study which showed increased workload or nursing staff numbers significantly affected patient outcome like the length of stay and complication(36). Even though the practical meaning of this result needs further experience on staffing and time to discharge, it is important to consider recommendations of safe staffing to maintain patient safety and to reduce the length of hospitalization(38).

\section{Conclusion}

Median time to discharge from the medical ward was longer than from surgical wards. Generally, surgical admitted patients were more prone to stay in hospital than medical admitted patients. Increased age, combined non-communicable, maternal and prenatal condition admission, increasing nurse patients' ratio, and higher medication adherence decreased discharge time. presence of family care, lab, and radiological result delay, presence of comorbidity, admission from ICU, off-hour admission, surgical wards admission increased discharge time. 


\section{Limitation Of The Study}

Although the range of participants was broad: men and women, from children to elderly people were included in this study, the generalizability of duration of discharge in other hospitals in the country should account for other possible confounders like staffing, standards of hospitals. Because of failing to measure the severity of disease due to a lack of validated/standard tools to study setting, the generalizability of these results was limited to the broad categorization of patients into wards. But the research question was answered moderately by considering the difference grouping into wards. And also faced with problems to measure clinical investigation turnaround time for wards admitted patients as predicted, resulting in difficulty to measure the specific time of investigations result in arrival time. Thus, this study considered the number of days, investigations results take to arrive at the patient's bedside to consider its effect on time to discharge. In addition, similar topic researches were limited in Ethiopia and Africa to compare the results with an expectation from previous studies.

\section{Recommendation}

Administrators of the hospital should have emphasized good communication of respective specialty to avoid delay of test results, and also practitioner should consider on teaching the benefit of medication adherence to the patients to reduce hospital stay. Further research is needed to establish a closer prediction of discharge time using disease severity and other hidden confounders.

\section{List Of Abbreviations}




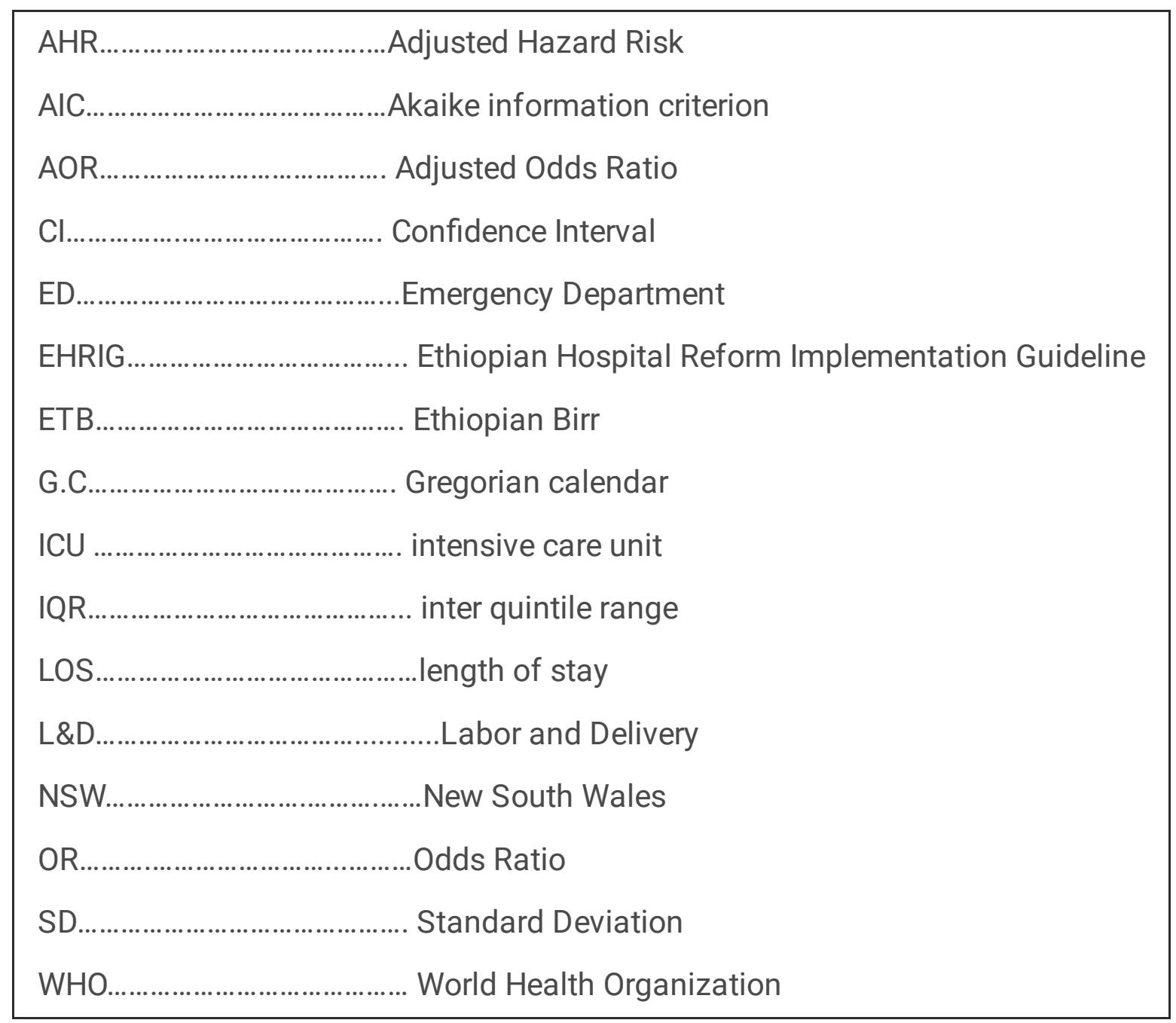

\section{Declarations}

\section{Ethics approval and consent to participate}

Ethical clearance which confirms that all methods were carried in accordance with relevant guidelines and regulations were approved by and obtained from Bahir Dar University, College of medicine and health science Institutional Review Board in February 2020 (No: 00116/2020). A formal letter of cooperation was written from the University of Bahir Dar ethical clearance committee to Felege Hiwot comprehensive specialized hospital.

Furthermore, written informed consent was obtained from each study participant by providing information about the purpose and benefit of the study. The participants were told about their right to withdraw from the study at any time. Confidentiality of the information was maintained throughout the study period by using anonymity identifiers.

\section{Consent for publication}

Not applicable 


\section{Availability of data and materials}

The datasets analyzed during this study were available from the corresponding author on reasonable request. Making the data public would result in stolen or misused by others, so upon reasonable request, the correspondence author will share it.

\section{Competing interests}

The authors declare that they have no competing interests.

\section{Funding}

Jigjiga University is only the funding body.

\section{Authors' contributions}

A.H, G.K, and T.G designed the study prepared the proposal analyzed the data interpreted the result, and wrote the draft. All authors read and approved the final manuscript.

\section{Acknowledgments}

we would like to express our heartfelt gratefulness towards advisors for their constructive comments and suggestions, and also to data collectors, respondents, Bahir Dar University and Felege Hiwot comprehensive specialized hospital.

\section{Authors' information}

${ }^{1}$ Jigjiga University college of medicine and health science, department of public health, Jigjiga, Ethiopia.

${ }^{2}$ Dire-Dawa university college of medicine and health science, department of public health, Dire Dawa, Ethiopia.

\section{References}

1. Khosravizadeh O, Vatankhah S, Bastani P, Kalhor R, Alirezaei S, Doosty F. Factors affecting the length of stay in teaching hospitals of a middle-income country. Electronic physician. 2016;8(10):3042-7.

2. Minister Of Health. Ethiopian Hospital Services Transformation Guidelines In: INITIATIVE EHM, editor. first edition ed. Addis ababa2016. p. 19-8. 
3. Health MO. Ethiopian hospital reform implementation guidelines. In: initiative Ehm, editor. Addis Ababa: Minister Of Health;; 2010.

4. Minister Of Health. Hospital Performance Monitoring and Improvement Manual. In: Directorate HSQ, editor. Second Edition ed. Addis ababa2017.

5. Garling P. Final Report of the Special Commission of Inquiry: Acute Care in NSW Public Hospitals, 2008. State of NSW through the Special Commission of Inquiry: Acute Care Services in New South Wales Public Hospitals Retrieved January. 2008;16:2011.

6. Richardson DB. The access-block effect: relationship between delay to reaching an inpatient bed and inpatient length of stay. Medical Journal of Australia. 2002;177(9):492-5.

7. Negari KG, Rodamo KM, Hirigo AT. Factors associated with the length of stay in emergency departments in Southern-Ethiopia. BMC research notes. 2019;12(1):239.

8. FMoH E. Health Sector Transformation Plan. HSTP 2015/16-2019/20. August; 2015.

9. Cameron PA. What is the bed availability gap underlying chronic emergency department access block? Canadian Journal of Emergency Medicine. 2019;21(2):165-6.

10. Lim S, Doshi V, Castasus B, Lim J, Mamun K. Factors causing delay in discharge of elderly patients in an acute care hospital. Annals-Academy of Medicine Singapore. 2006;35(1):27.

11. Leroyer A, Bedu A, Lombrail P, Desplanques L, Diakite B, Bingen E, et al. Prolongation of hospital stay and extra costs due to hospital-acquired infection in a neonatal unit. Journal of Hospital Infection. 1997;35(1):37-45.

12. Vegasi AA, Jodra VM, García ML. Nosocomial infection in surgery wards: a controlled study of increased duration of hospital stays and direct cost of hospitalization. European journal of epidemiology. 1993;9(5):504-10.

13. Victor CR, Healy J, Thomas A, Seargeant J. Older patients and delayed discharge from hospital. Health \& social care in the community. 2000;8(6):443-52.

14. Rojas-García A, Turner S, Pizzo E, Hudson E, Thomas J, Raine R. Impact and experiences of delayed discharge: A mixed-studies systematic review. Health Expectations. 2018;21(1):41-56.

15. Fekadu A, Desta M, Alem A, Prince M. A descriptive analysis of admissions to Amanuel Psychiatric Hospital in Ethiopia. Ethiopian Journal of Health Development. 2007;21(2):173-8.

16. REPRESENT H. Reducing patient time in the emergency department. The Medical Journal of Australia. 2003;179(10):516-7.

17. Taylor DM, Bennett D, Cameron PA. A paradigm shift like care provision in emergency departments. Emergency Medicine Journal. 2004;21(6):681-4.

18. Langhan TS. Do elective surgical and medical admissions impact emergency department length of stay measurements? Clinical and Investigative Medicine. 2007:E177-E82.

19. Forster AJ, Stiell I, Wells G, Lee AJ, Van Walraven C. The effect of hospital occupancy on emergency department length of stay and patient disposition. Academic Emergency Medicine. 2003;10(2):12733. 
20. HOSPITAL ANNUAL REPORT OF 2011EFY. Bahirdar: Felege hiwot comprehensive specialized hospital; 2011 EFY.

21. Shaffer EE, Pham A, Woldman RL, Spiegelman A, Strassels SA, Wan GJ, et al. Estimating the Effect of Intravenous Acetaminophen for Postoperative Pain Management on Length of Stay and Inpatient Hospital Costs. Advances in Therapy. 2016;33(12):2211-28.

22. Kishi Y, Meller WH, Kathol RG, Swigart SE. Factors affecting the relationship between the timing of psychiatric consultation and general hospital length of stay. Psychosomatics. 2004;45(6):470-6.

23. Tashkandy MA, Gazzaz ZJ, Farooq MU, Dhafar KO. Reasons for delay in inpatient admission at an emergency department. J Ayub Med Coll Abbottabad. 2008;20(1):38-42.

24. Ambaw AD, Alemie GA, Mengesha ZB. Adherence to antihypertensive treatment and associated factors among patients on follow-up at University of Gondar Hospital, Northwest Ethiopia. BMC public health. 2012;12(1):282.

25. Rosen OZ, Fridman R, Rosen BT, Shane R, Pevnick JM. Medication adherence as a predictor of 30day hospital readmissions. Patient preference and adherence. 2017;11:801.

26. Gigantesco A, De Girolamo G, Santone G, Miglio R, Picardi A. Long-stay in short-stay inpatient facilities: risk factors and barriers to discharge. BMC Public Health. 2009;9(1):306.

27. Martin S, Smith P. Explaining variations in inpatient length of stay in the National Health Service. Journal of Health Economics. 1996;15(3):279-304.

28. Jasinarachchi KH, Ibrahim IR, Keegan BC, Mathialagan R, McGourty JC, Phillips JR, et al. Delayed transfer of care from NHS secondary care to primary care in England: its determinants, effect on hospital bed days, the prevalence of acute medical conditions and deaths during the delay, in older adults aged 65 years and over. BMC geriatrics. 2009;9(1):4.

29. Wright S, Verouhis D, Gamble G, Swedberg K, Sharpe N, Doughty R. Factors influencing the length of hospital stay of patients with heart failure. European Journal of Heart Failure. 2003;5(2):201-9.

30. Rochon PA, Katz JN, Morrow LA, McGlinchey-Berroth R, Ahlquist MM, Sarkari M, et al. Comorbid illness is associated with survival and length of hospital stay in patients with chronic disability: a prospective comparison of three comorbidity indices. Medical care. 1996:1093-101.

31. Hood SR, Giazzon AJ, Seamon G, Lane KA, Wang J, Eckert GJ, et al. Association between medication adherence and the outcomes of heart failure. Pharmacotherapy: The Journal of Human Pharmacology and Drug Therapy. 2018;38(5):539-45.

32. Silva SAd, Valácio RA, Botelho FC, Amaral CFS. Reasons for discharge delays in teaching hospitals. Revista de saude publica. 2014;48(2):314-21.

33. Vetrano DL, Landi F, De Buyer SL, Carfi A, Zuccalà G, Petrovic M, et al. Predictors of length of hospital stay among older adults admitted to acute care wards: a multicentre observational study. European journal of internal medicine. 2014;25(1):56-62.

34. Liew D, Liew D, Kennedy MP. Emergency department length of stay independently predicts excess inpatient length of stay. Med J Aust. 2003;179(10):524-6. 
35. Shanley LA, Lin H, Flores G. Factors associated with length of stay for pediatric asthma hospitalizations. Journal of Asthma. 2015;52(5):471-7.

36. Amaravadi RK, Dimick JB, Pronovost PJ, Lipsett PA. ICU nurse-to-patient ratio is associated with complications and resource use after esophagectomy. Intensive care medicine. 2000;26(12):185762.

37. Thungjaroenkul P, Cummings GG, Embleton A. The impact of nurse staffing on hospital costs and patient length of stay: a systematic review. Nursing Economics. 2007;25(5):255.

38. Education LN. The Importance of the Optimal Nurse-to-Patient Ratio. Lippincott Nursing Education Blog Last accessed April. 2016;28:2020.

\section{Figures}

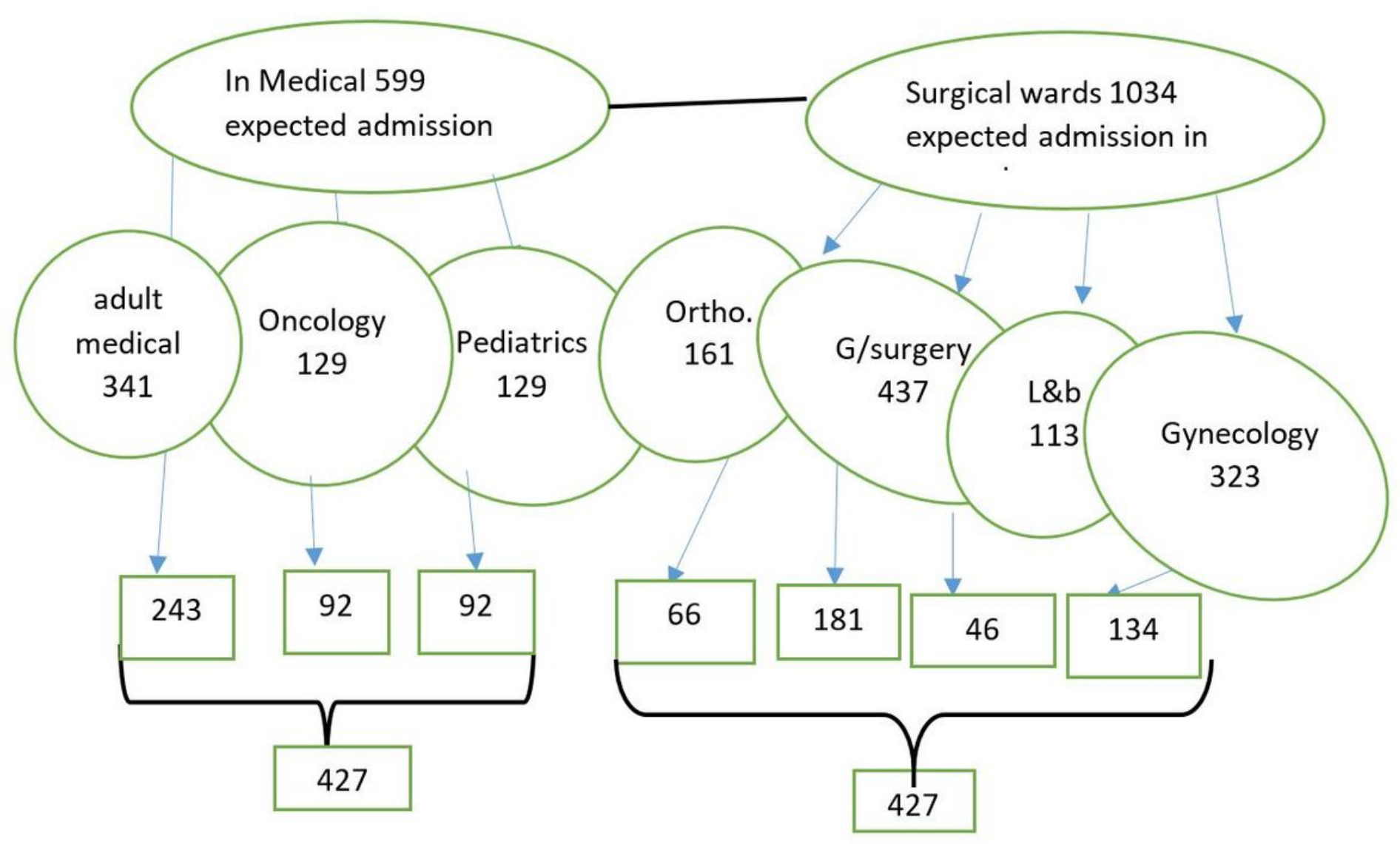

Figure 1

sampling procedure for study participants from two major wards in Felege Hiwot compressive specialized hospitals in 2020 


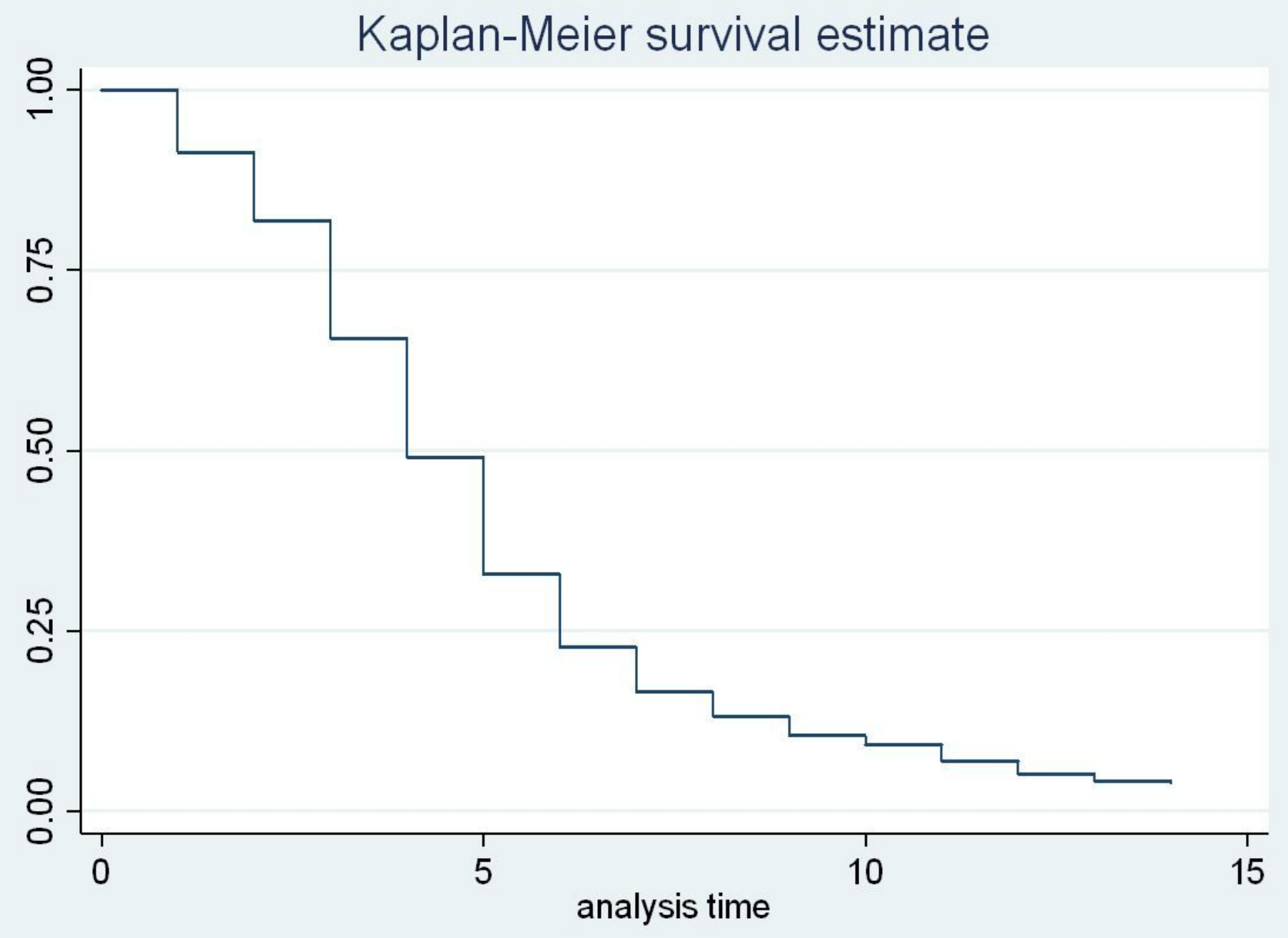

Figure 2

Kaplan Meier curve showing survival of discharge in Felege Hiwot compressive specialized hospital, 2020 


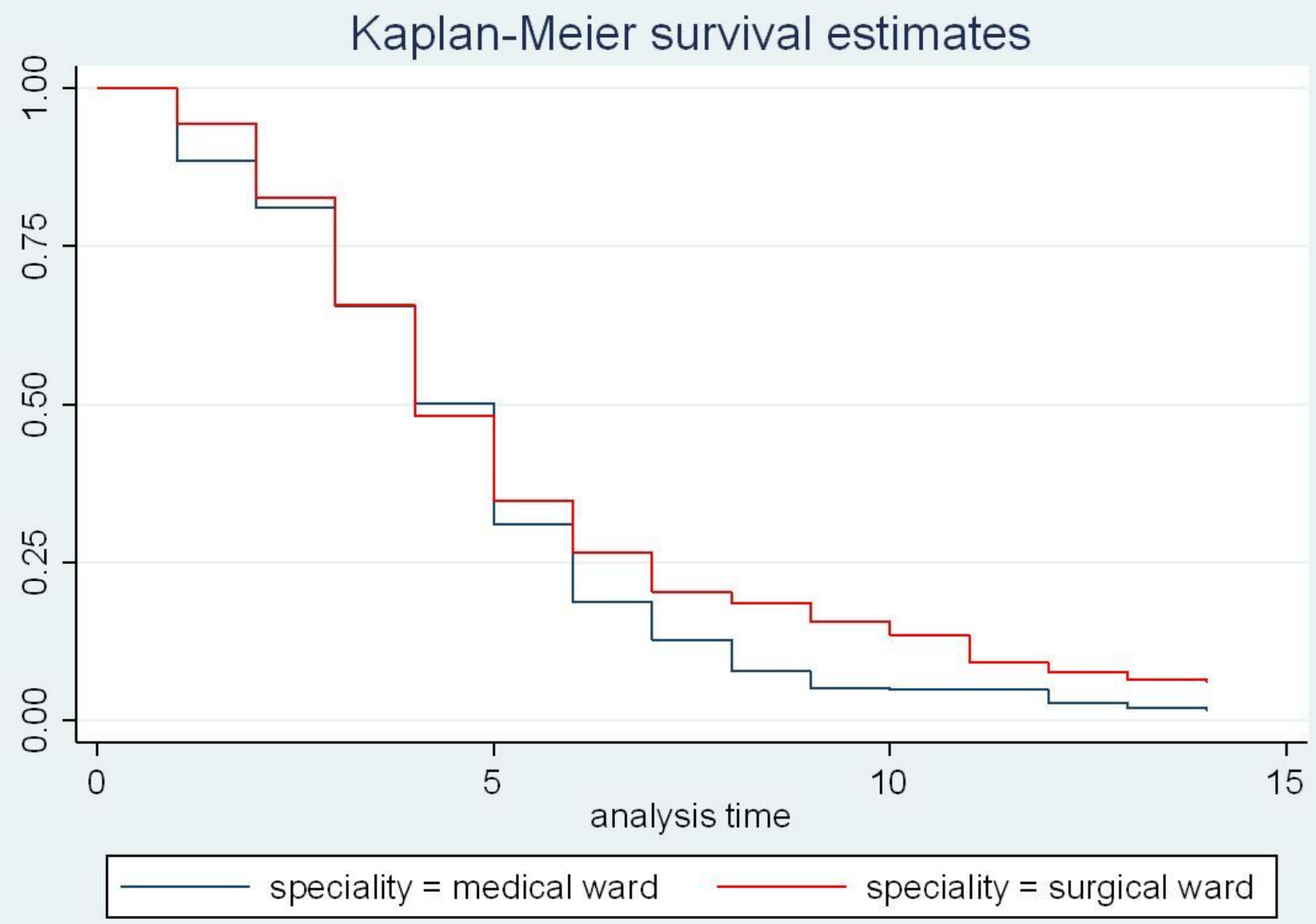

Figure 3

Kaplan Meier curve of medical and surgical ward admitted discharges in Felege Hiwot compressive specialized hospital, 2020 


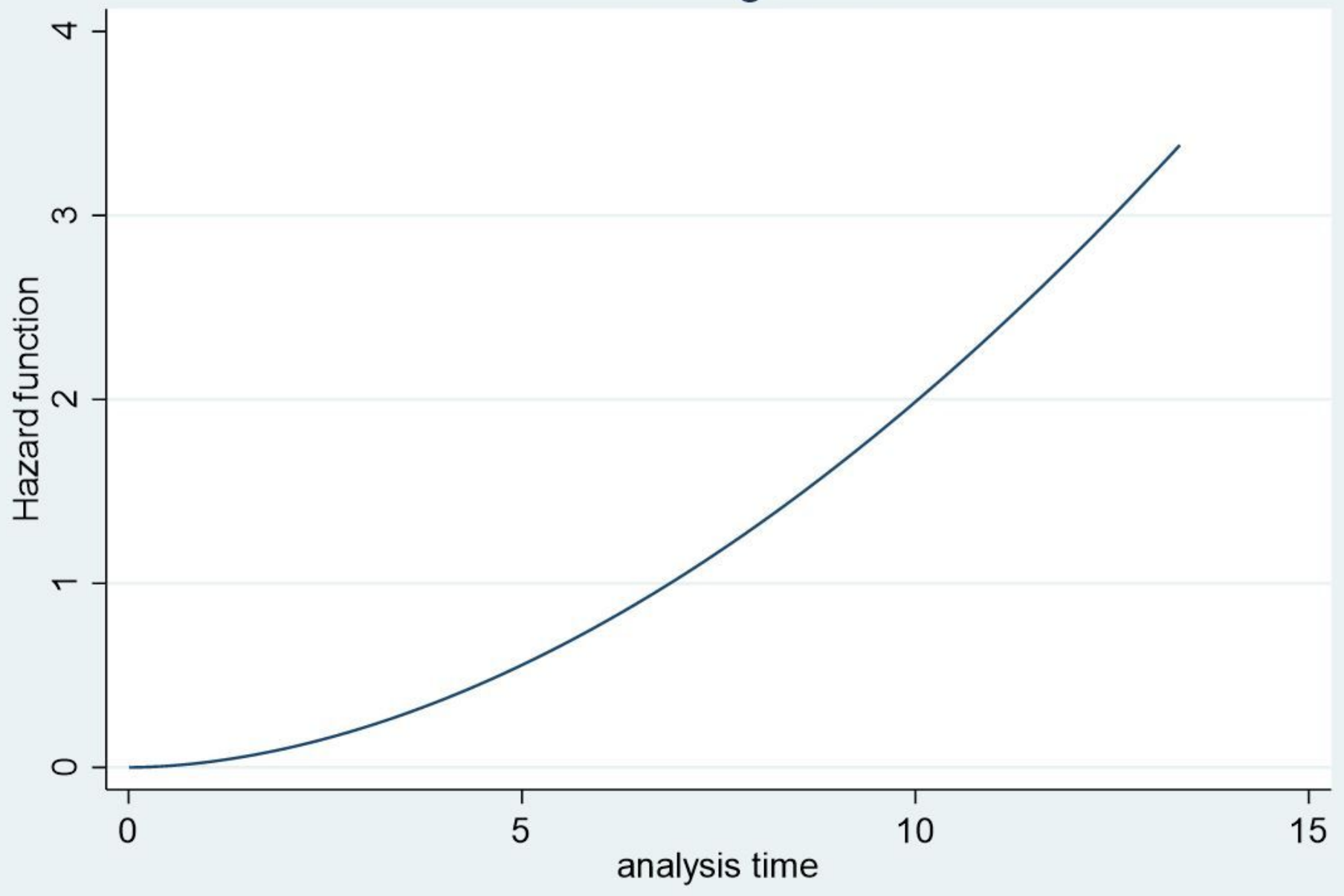

Figure 4

A plot of hazard function from Weibull regression to show model fitness 


\section{Cumulative hazard vs Cox-Snell}

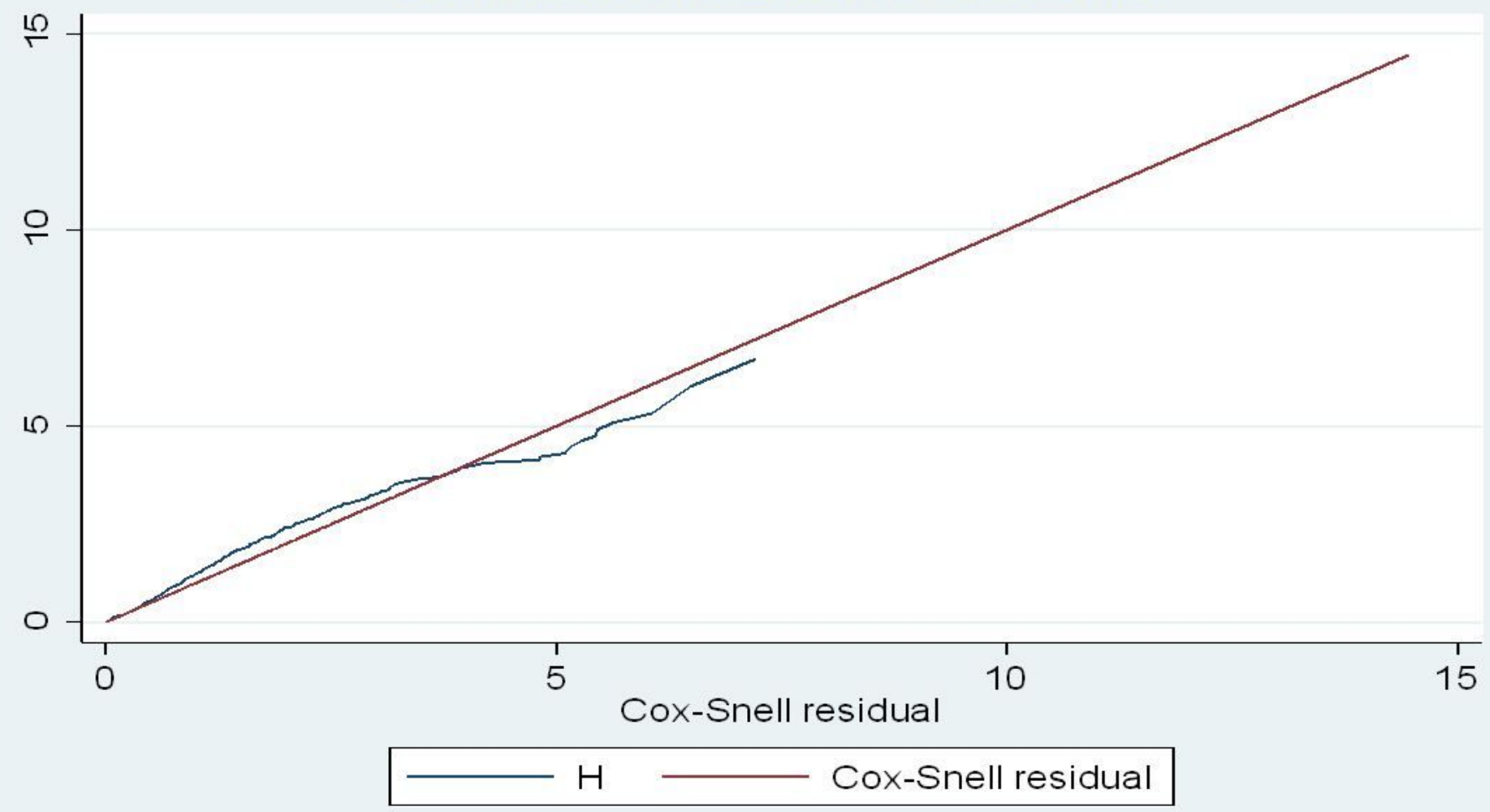

\section{Figure 5}

The goodness of fit Plot of cumulative hazard function from Weibull regression 